\title{
BMJ Open Antibiotic susceptibility profiles of ocular and nasal flora in patients undergoing cataract surgery in Taiwan: an observational and cross- sectional study
}

\author{
Yun-Hsuan Lin, ${ }^{1}$ Yu-Chuan Kang, ${ }^{1}$ Chiun-Ho Hou, ${ }^{1,2}$ Yhu-Chering Huang, ${ }^{2,3}$ \\ Chih-Jung Chen, ${ }^{2,3}$ Jwu-Ching Shu, ${ }^{4}$ Pang-Hsin Hsieh, ${ }^{2,5}$ Ching-Hsi Hsiao ${ }^{1,2}$
}

To cite: Lin Y-H, Kang Y-C, Hou C-H, et al. Antibiotic susceptibility profiles of ocular and nasal flora in patients undergoing cataract surgery in Taiwan: an observational and cross-sectional study. BMJ Open 2017;7:e017352. doi:10.1136/ bmjopen-2017-017352

- Prepublication history for this paper is available online. To view these files please visit the journal online (http://dx.doi org/10.1136/bmjopen-2017017352).

Received 18 April 2017 Revised 28 June 2017 Accepted 12 July 2017

\section{CrossMark}

${ }^{1}$ Department of Ophthalmology, Chang Gung Memorial Hospital, Keelung, Keelung, Taiwan

${ }^{2}$ College of Medicine, Chang Gung University, Kwei-Shan, Taiwan

${ }^{3}$ Division of Paediatric Infectious Diseases, Department of Pediatrics, Chang Gung Memorial Hospital, Keelung, Taiwan

${ }^{4}$ Department of Medical Biotechnology and Laboratory Science, College of Medicine, Chang Gung University, KweiShan, Taiwan

${ }^{5}$ Department of Orthopaedic Surgery, Chang Gung Memorial Hospital, Kwei-shan, Taiwan

Correspondence to

Dr Ching-Hsi Hsiao;

hsiao.chinghsi@gmail.com

\section{ABSTRACT}

Objective To investigate the conjunctival and nasal flora and the antibiotic susceptibility profiles of isolates from patients undergoing cataract surgery.

Design Observational and cross-sectional study.

Setting A single-centre study in Taiwan.

Participants 128 consecutive patients precataract surgery.

Primary and secondary outcome measures methods Conjunctival and nasal cultures were prospectively obtained from 128 patients on the day of cataract surgery before instillation of ophthalmic solutions in our hospital. Isolates and antibiotic susceptibility profiles were identified through standard microbiological techniques. Participants were asked to complete a questionnaire on healthcare-associated factors. Results The positive culture rate from conjunctiva was $26.6 \%$, yielding 84 isolates. Coagulase-negative Staphylococci were the most commonly isolated organisms (45.2\%), and $35 \%$ of staphylococcal isolates were methicillin-resistant. Among staphylococcal isolates, all were susceptible to vancomycin, and $75 \%$ $82.5 \%$ were susceptible to fluoroquinolones. Methicillinresistant isolates were significantly less susceptible than their methicillin-sensitive counterparts to tobramycin, the most commonly used prophylactic antibiotic in our hospital ( $28.6 \%$ vs $69.2 \%$; $p=0.005$ ). The positive culture rate from nares for Staphylococcus aureus was $21.9 \%$, and six isolates were methicillin-resistant. No subjects had $S$. aureus colonisation on conjunctiva and nares simultaneously. There were no associated risk factors for colonisation of methicillin-resistant Staphylococci.

Conclusion The most common conjunctival bacterial isolate of patients undergoing cataract surgery was coagulase-negative Staphylococci in Taiwan. Because of predominant antibiotic preferences and selective antibiotic pressures, Staphylococci were more susceptible to fluoroquinolones but less to tobramycin than in other reports. Additionally, methicillin-resistant Staphylococci exhibited co-resistance to tobramycin but not to fluoroquinolones.

\section{Strengths and limitations of this study}

- This observational and cross-sectional study was to investigate the conjunctival and nasal flora and the antibiotic susceptibility profiles of isolates from patients undergoing cataract surgery in Taiwan where such information is limited.

- Our results delineate methicillin-resistant Staphylococci co-resistance to tobramycin but not to fluoroquinolones in Taiwan.

- Associated risk factors for methicillin-resistant Staphylococci colonisation were not found in current study.

- The culture rate from conjunctiva was lower than that obtained from other studies.

- Small sample sizes in the current study may limit statistical significance.

\section{INTRODUCTION}

Cataract surgery is one of the most common surgical procedures performed by ophthalmologists. Endophthalmitis is a rare but devastating complication of cataract surgery. Gram-positive pathogens are responsible for $60 \%-80 \%$ of endophthalmitis; among them, coagulase-negative Staphylococci (CNS) are the most frequent isolates. ${ }^{1-3}$ The source of pathogens for endophthalmitis is mainly the ocular surface of the patients. Additionally, nasal mucosa serves as a reservoir of organisms for the conjunctiva and nasolacrimal system. Speaker et al demonstrated that organisms isolated from the vitreous of endophthalmitis were genetically indistinguishable from those derived from the eyelids, conjunctiva and noses in $82 \%$ of cases. ${ }^{4}$ Thus, evaluation of the flora and their drug susceptibility may serve as the success of empiric perioperative antibiotic use. However, the risk and success factors may vary across geographic regions 
and over periods of time..$^{5-7}$ So, local epidemiological data must be established, and periodic surveys must be conducted to serve as references for clinicians.

Methicillin-resistant Staphylococcus sp are not only resistant to methicillin, but also to all $\beta$-lactam antibiotics. Additionally, methicillin resistance might also be related to resistance to other classes of antibiotics, which may limit treatment options. Methicillin-resistant Staphylococcus is a major concern for global public health. Recently, studies have focused on endophthalmitis caused by methicillin-resistant Staphylococcus. ${ }^{8-11}$ Some notable features of methicillin-resistant Staphylococcus-associated endophthalmitis are as follows: a rise in the reported incidence rate, ${ }^{81011}$ an association with poor visual outcome ${ }^{811}$ and an association with resistance to fluoroquinolones. ${ }^{811}$ Methicillin-resistant Staphylococcus aureus (MRSA) colonisation is an important risk factor for subsequent MRSA- related infections in other fields. ${ }^{12} 13$ Identifying patients with a high risk of developing methicillin-resistant Staphylococcus infections may be helpful in preventing severe complications from cataract surgery.

To effectively prescribe empiric antibiotics, a profile of likely pathogens in a local community is required. However, information regarding bacterial colonisation on ocular surfaces in Taiwan is limited. Additionally, methicillin-resistant Staphylococcus is prevalent in Taiwan. However, it is unknown whether these organisms colonise the ocular surface. Therefore, we conducted a study to investigate the conjunctival and nasal flora and the antibiotic susceptibility profiles of the isolates from patients undergoing cataract surgery at Chang Gung Memorial Hospital (CGMH). Furthermore, we focused on methicillin-resistant Staphylococcus sp and explored the associated risk factors. We intend to provide baseline data to help local clinicians choose appropriate antibiotic prophylaxes for their patients.

\section{METHODS}

\section{Study participants and data collection}

From 1 August 2012 to 31 July 2013, we enrolled patients $>18$ years old who were scheduled for elective cataract surgery. Exclusion criteria included pregnancy, use of oral or topical antibiotics within 3 months before surgery, and active ocular infection or conjunctivitis. The nature and procedures of the study were fully explained to all participants, and informed consent was obtained before recruitment. The Institutional Review Board of CGMH approved the study.

Participants were asked to complete a questionnaire on healthcare-associated factors. Questions regarding hospitalisation, surgery, dialysis, duration of stay in healthcare facilities, catheter use, use of immunosuppressive medications and antimicrobials, comorbid medical conditions (cardiovascular disease, liver disease, asthma, chronic obstructive pulmonary disease, renal failure, HIV, malignancy and diabetes mellitus), sociodemographic data (alcohol abuse, homelessness, close contact with children
$<5$ years and close contact with children who attend daycare) and ocular history (ocular disease, surgery, contact lens use and topical medication) were included.

\section{Sampling of bacteria}

Prior to the preoperative instillation of topical medication, cultures were obtained from the bilateral lower conjunctival sac using a sterile calcium alginate. Contact with eyelids and eyelashes was avoided, and the cultures were immediately inoculated onto 5\% sheep blood and chocolate agar plates and sent to the CGMH laboratory. Separate cultures were obtained from both eyes. All culture plates were incubated at $37^{\circ} \mathrm{C}$ for 3 days and observed. Cultures were deemed positive if colony-forming units were observed. Isolates were identified using standard microbiological protocols. Nasal cultures were taken with swab and transport system and sent for cultures; only $S$. aureus were identified and isolated, if present.

\section{Bacterial susceptibility testing}

To determine susceptibility, the Kirby-Bauer disc diffusion technique was conducted in strict accordance with the guidelines of the National Committee for Clinical Laboratory Standards Institute. Selected antibiotics were tested on different micro-organisms. In addition, an E-test (BioMerieux SA, Marcy-I'Etoile, France) was used to determine the susceptibility to tobramycin and fluoroquinolones including ciprofloxacin, levofloxacin, gatifloxacin and moxifloxacin, which were not included in the antibiotic susceptibility profiles for gram-positive bacteria in our microbiology laboratory. Cefoxitin resistance was considered equivalent to methicillin resistance. Resistance was defined as being 'resistant' or 'intermediate' in susceptibility.

\section{Data analysis}

Descriptive statistics (eg, mean) were calculated for case characteristics. Group comparisons were performed using the $\mathrm{X}^{2}$ test and the Fisher exact test. A p value $<0.05$ was considered significant.

\section{RESULTS}

\section{Baseline characteristics}

In total, 128 patients who underwent cataract surgery were recruited in the study. The average age of the patients was 68.3 years, and $60(46.9 \%)$ patients were men, $79(61.7 \%)$ had underlying systemic diseases and $53(41.4 \%)$ had at least one of the aforementioned healthcare-associated factors. Postoperatively, all the patients were placed on tobramycin/dexamethasone solution (Alcon, Puurs, Belgium) four times daily. No patient developed endophthalmitis during the follow-up period.

\section{Bacterial cultures}

Fifty-four patients had positive culture results from the conjunctiva. Among them, 14 patients had bilaterally positive culture results. The positive culture rate from 


\begin{tabular}{lll}
\hline $\begin{array}{l}\text { Table } 1 \\
\text { patients }\end{array}$ & Microbial isolates from the conjunctiva of cataract \\
\hline Conjunctival isolates & $\begin{array}{l}\text { Number } \\
\text { (n=84) }\end{array}$ & $\begin{array}{l}\text { Percentage } \\
\text { (\%) }\end{array}$ \\
\hline Gram-positive bacteria & 76 & 91.7 \\
\hline Staphylococcus epidermidis & 14 & 16.7 \\
CNS (other) & 24 & 28.6 \\
\hline S. aureus & 2 & 2.4 \\
\hline Gram-positive bacilli* & 29 & 34.5 \\
\hline Streptococcus sp & 3 & 3.6 \\
\hline Enterococcus faecium & 2 & 2.4 \\
\hline Micrococcus sp & 2 & 2.4 \\
\hline Gram-negative bacteria & 7 & 8.3 \\
\hline Pseudomonas sp & 4 & 4.8 \\
\hline Moraxella species & 1 & 1.2 \\
\hline Other gram-negative species & 2 & 2.4 \\
\hline Fungus & $\mathbf{1}$ & 1.2 \\
\hline Yeast-like & 1 & 1.2 \\
\hline Gram-positive baciling & & 2 \\
\hline
\end{tabular}

${ }^{*}$ Gram-positive bacilli included Lactobacillus sp, Corynebacteria $\mathrm{sp}$, Listeria sp and Bacillus sp.

CNS, coagulase-negative staphylococcus.

conjunctiva was $26.6 \%$ ( 68 of 256 eyes). Among the 84 isolates, $76(91.7 \%)$ were gram-positive bacteria, 7 (8.3\%) were gram-negative bacteria and $1(1.2 \%)$ was fungus. CNS was the most predominant group of bacterial organisms (38 isolates, $45.2 \%$ of the isolates), and more than one-third (14 isolates) was S. epidermidis. S. aureus isolates were only observed in two eyes, and both were methicillin-sensitive (table 1).

Among Staphylococcus sp from conjunctiva, 35\% (14 of 40) of staphylococcal isolates were methicillin-resistant. A comparison between the baseline demographic characteristics of patients colonised with methicillin-sensitive and methicillin-resistant Staphylococcus sp revealed no significantly associated risk factors between the two groups (table 2).

The positive culture rate from nares for $S$. aureus was $21.9 \%$ (28 of 128), and six isolates were methicillin-resistant. None of the study subjects had $S$. aureus colonisation on both conjunctiva and nares.

\section{Antibiotic susceptibility of Staphylococcus species}

Table 3 illustrates the antibiotic susceptibility of staphylococcal isolates, including methicillin-resistant and sensitive strains. In total, Staphylococcus sp demonstrated the least susceptibility to penicillin $(22.5 \%, 9$ of 40$)$, followed by tobramycin $(55 \%, 22$ of 40$)$. All staphylococcal isolates were sensitive to vancomycin and teicoplanin. Regarding the fluoroquinolones, the rates of susceptibility to ciprofloxacin, levofloxacin, gatifloxacin and moxifloxacin were $80 \%, 80 \%, 75 \%$, and $82.5 \%$, respectively.

In comparison to methicillin-sensitive staphylococcal isolates, methicillin-resistant isolates were significantly
Table 2 Comparison of demographics and characteristics of $26 \mathrm{MSS}$ sp and $14 \mathrm{MRS}$ sp isolated from conjunctiva

\begin{tabular}{lllc}
\hline & $\begin{array}{l}\text { MSS } \\
(\mathbf{n = 2 6 )} \\
\text { No. }\end{array}$ & $\begin{array}{l}\text { MRS } \\
(\mathbf{n = 1 4}) \\
\text { No. }\end{array}$ & p Value $^{*}$ \\
\hline Gender (men) & 12 & 7 & 0.816 \\
\hline Age (years) & $70.3 \pm 10.4$ & $71.1 \pm 9.1$ & 0.218 \\
$\begin{array}{l}\text { Healthcare-associated } \\
\text { factors }\end{array}$ & 5 & 4 & 0.694 \\
$\begin{array}{l}\text { Systemic history } \\
\quad \text { Skin disease }\end{array}$ & 3 & 1 & $>0.999 \dagger$ \\
\hline Liver disease & 2 & 0 & $0.533 \dagger$ \\
\hline $\begin{array}{l}\text { Diabetes mellitus } \\
\text { Heart disease }\end{array}$ & 9 & 5 & $>0.999 \dagger$ \\
\hline $\begin{array}{l}\text { Chronic kidney disease } \\
\text { Hypertension }\end{array}$ & 5 & 1 & $0.399 \dagger$ \\
\hline $\begin{array}{l}\text { Ocular history } \\
\text { Ocular surgery }\end{array}$ & 16 & 0 & $0.143 \dagger$ \\
\hline Personal history & 1 & 1 & $0.730 \dagger$ \\
\hline $\begin{array}{l}\text { Systemic antibiotic } \\
\text { history }\end{array}$ & 0 & 0 & $>0.999 \dagger$ \\
\hline $\begin{array}{l}\text { Alcoholism } \\
\text { Live with kids }\end{array}$ & 1 & 0 & $>0.999 \dagger$ \\
\hline
\end{tabular}

*Student's t-test for age comparison, $\mathrm{X}^{2}$ test for others.

$\dagger \mathrm{X}^{2}$ not be a valid test owing to a low $\mathrm{n}$ umber; Fisher exact test was used.

MRS, methicillin-resistant Staphylococcus; MSS, methicillinsusceptible Staphylococcus.

less susceptible to sulfamethoxazole-trimethoprim (64.3\% vs $96.2 \% ; \mathrm{p}=0.014)$ and tobramycin $(28.6 \%$ vs $69.2 \% ; \mathrm{p}=0.005)$.

All $S$. aureus isolated from nares were susceptible to sulfamethoxazole-trimethoprim, teicoplanin and vancomycin. MRSA isolates were less susceptible to clindamycin and erythromycin than were methicillin-sensitive $S$. aureus ( $\mathrm{p}=0.001$ and 0.007 , respectively; table 4 ).

\section{DISCUSSION}

Although substantial evidence exists regarding the efficacy and safety of intracameral antibiotics, topical antibiotics are still the predominant form of prophylaxis for postoperative endophthalmitis employed by surgeons. ${ }^{14}$ Understanding the spectrum of the ocular flora and their antibiotic susceptibility in various geographic locales can assist clinicians in optimising prophylactic antibiotic treatments. We herein present results from a cohort of patients who underwent cataract surgery at our institution. Our findings indicate that CNS is the most common organism isolated from conjunctiva, and that more than one-third of CNS isolates are methicillin-resistant; in Taiwan, methicillin-resistant Staphylococci exhibits multidrug resistance 
Table 3 Comparison of antibiotic susceptibilities of 26 MSS sp and 14 MRS sp isolated from conjunctiva

\begin{tabular}{lllll}
\hline \multicolumn{5}{c}{ Susceptible Strains } \\
\cline { 2 - 5 } & Total (n=40)N (\%) & MSS (n=26)N (\%) & MRS (n=14)N (\%) & $\mathbf{p ~ V a l u e}^{*}$ \\
\hline Clindamycin & $35(87.5)$ & $23(88.5)$ & $12(85.7)$ & $>0.999 \dagger$ \\
Erythromycin & $27(67.5)$ & $17(65.4)$ & $10(71.4)$ & $>0.999 \dagger$ \\
\hline Penicillin & $9(22.5)$ & $9(34.6)$ & $0(0)$ & $0.016 \dagger$ \\
Sulfamethoxazole-trimethoprim & $34(85)$ & $25(96.2)$ & $9(64.3)$ & $0.014 \dagger$ \\
Teicoplanin & $40(100)$ & $26(100)$ & $14(100)$ & \\
Vancomycin & $40(100)$ & $26(100)$ & $14(100)$ & \\
Ciprofloxacin & $32(80)$ & $21(80.8)$ & $11(78.6)$ & $0.650 \dagger$ \\
Gatifloxacin & $30(75)$ & $20(76.9)$ & $10(71.4)$ & $0.433 \dagger$ \\
Levofloxacin & $32(80)$ & $21(80.8)$ & $11(78.6)$ & $0.650 \dagger$ \\
Moxifloxacin & $33(82.5)$ & $22(84.6)$ & $11(78.6)$ & $0.337 \dagger$ \\
Tobramycin & $22(55)$ & $18(69.2)$ & $4(28.6)$ & 0.005 \\
\hline
\end{tabular}

*Student's t-test for age comparison, $\mathrm{X}^{2}$ test for others.

$\dagger \mathrm{X}^{2}$ not be a valid test owing to a low $\mathrm{n}$ umber; Fisher exact test was used.

MRS, methicillin-resistant Staphylococcus; MSS, methicillin-susceptible Staphylococcus.

to sulfamethoxazole-trimethoprim and tobramycin but not to fluoroquinolones.

The culture rate from conjunctiva was $26.6 \%$ in this study. To improve culture yield, we inoculated the conjunctival swab samples directly into culture media instead of transport media. Our culture rate was lower than that obtained from other studies ${ }^{15-18}$ but moderately higher than in a previous study conducted at CGMH from 2002 to $2008(18 \%) .{ }^{19}$ By contrast, the culture rate from nares for $S$. aureus was consistent with that of previous studies. ${ }^{20-23}$ Insufficient rotation of the cotton swab on the conjunctiva, lengthy shipping time and culture conditions may have contributed to the low culture yield, although the specific cause of the low conjunctival culture rate is uncertain.

Normal conjunctival flora is predominantly composed of gram-positive bacteria and CNS, which were the most commonly isolated organisms in our study. This finding agrees with previous reports wherein CNS represented the most commonly isolated bacteria from ocular surfaces of patients undergoing ophthalmic surgery ${ }^{18} 24$ and from patients with postoperative endophthalmitis. ${ }^{25} 26$

We then examined the antibiotic susceptibility of Staphylococcus sp, particularly methicillin-resistant isolates that might exhibit multidrug resistance and be associated with virulent infections. ${ }^{27}$ The rate of methicillin resistance among staphylococcal isolates was $35 \%$, in accordance with a reported rate ranging from $35 \%$ to $50 \%$ in numerous studies since 2000s. ${ }^{16} 182028-32$

Our methicillin-resistant staphylococcal isolates were significantly more resistant to certain antibiotics than their methicillin-sensitive counterparts, including sulfamethoxazole-trimethoprim and tobramycin. The 2005-2006 Ocular TRUST report indicated that MRSA isolates were more resistant to all classes of antibiotics than were MSSA, except for trimethoprim. ${ }^{6}$ A recently updated report by the Antibiotic Resistance Monitoring in Ocular Micro-organisms (ARMOR) indicated that methicillin-resistant isolates had a high probability of

Table 4 Comparison of antibiotic susceptibilities of 22 MSSA and 6 MRSA isolated from nares

\begin{tabular}{llllc}
\hline & \multicolumn{3}{l}{ Susceptible strains } & \\
\cline { 2 - 5 } & Total $(\mathbf{n = 2 8 ) \mathbf { N }}(\%)$ & MSSA (n=22)N (\%) & MRSA (n=6)N (\%) & p Value* $^{*}$ \\
\hline Clindamycin & $21(75.0)$ & $20(90.9)$ & $1(16.7)$ & $0.001 \dagger$ \\
Erythromycin & $19(67.9)$ & $18(81.8)$ & $1(16.7)$ & $0.007 \dagger$ \\
Penicillin & $2(7.1)$ & $2(9.1)$ & $0(0)$ & $>0.999 \dagger$ \\
Linezolid & $28(100)$ & $22(100)$ & $6(100)$ & \\
Sulfamethoxazole-trimethoprim & $28(100)$ & $22(100)$ & $6(100)$ & \\
Teicoplanin & $28(100)$ & $22(100)$ & $6(100)$ & \\
Vancomycin & $28(100)$ & $22(100)$ & $6(100)$ & \\
Tigecycline & $28(100)$ & $22(100)$ & $6(100)$ & \\
\hline
\end{tabular}

*Student's t-test for age comparison, $\mathrm{X}^{2}$ test for others.

$\dagger \mathrm{X}^{2}$ not be a valid test owing to a low number; Fisher exact test was used.

MRSA, methicillin-resistant Staphylococcus aureus; MSSA, methicillin-susceptible Staphylococcus aureus. 
concurrent resistance to fluoroquinolones, aminoglycosides and macrolides. ${ }^{33}$ Although reports vary, all the aforementioned studies (including this one) demonstrate that methicillin-resistant organisms exhibit stronger multidrug resistance than their methicillin-sensitive counterparts do.

We paid particular attention to the susceptibility of fluoroquinolones, the most common empiric and prophylactic antibiotics in the ophthalmic field. Growing fluoroquinolone resistance has been widely documented for ocular pathogens and common conjunctival flora. ${ }^{5} 18203031$ In the present study, the susceptibility rate of four tested fluoroquinolones for staphylococcal isolates from conjunctiva ranged from $75 \%$ to $82.5 \%$, which is slightly higher than that in earlier reports $(50 \%-80 \%) \%) .{ }^{16} 2030-32$ In contrast to some studies on staphylococcal isolates from normal conjunctival flora and ocular pathogens demonstrating that methicillin resistance can be a marker for fluoroquinolone resistance, ${ }^{811293033}$ we did not observe a significant difference in the susceptibility to fluoroquinolones between methicillin-sensitive and methicillin-resistant Staphylococci. Taiwan's National Health Insurance Administration reserves fluoroquinolones for the treatment of severe bacterial infections such as corneal ulcers (ie, clinicians may not use fluoroquinolones for prophylactic purposes or mild infections such as conjunctivitis), which might contribute to the relatively high susceptibility to fluoroquinolones in our isolates.

By contrast, the susceptibility rate of staphylococcal isolates to tobramycin in our study was $55 \%$, which is substantially lower than the rates described in recent literature, wherein $90 \%-100 \%$ of staphylococcal isolates were susceptible to tobramycin and gentamicin. ${ }^{16} 1820283132$ Additionally, methicillin-resistant staphylococci were more resistant to tobramycin than their methicillin-sensitive counterparts in our study $(71.4 \%$ vs $30.8 \%)$; the most recent ARMOR update delineated a similar finding, although a lower resistant rate to tobramycin was reported (14\% and $2 \%$ for methicillin-resistant and methicillin-sensitive CNS, respectively). ${ }^{33}$ Tobramycin is the most widely used prophylactic antibiotic for cataracts and other ocular surgeries at our institution and in Taiwan; exposure to subinhibitory concentrations of tobramycin may promote resistance. In sum, Staphylococci were more susceptible to fluoroquinolones but less susceptible to tobramycin in our study than in the majority of previous reports. ${ }^{16} 1820283132$ This may be caused by antibiotic selective pressure (ie, bacteria exposed to certain antibiotics causes emergence of resistant strains). Furthermore, the prevalence of antibiotic use may vary in different regions, which can cause different susceptibility patterns of the isolates. These results highlight the necessity of establishing local epidemiological information.

Our results delineate the tobramycin and multidrug resistance of Staphylococci in Taiwan. Tobramycin may not be an effective first-line effort for ophthalmic prophylaxis, particularly for methicillin-resistant Staphylococci in
Taiwan. Preoperative sterilisation of ocular surface with $5 \%-10 \%$ povidone-iodine is effective in the reduction of bacterial counts on the conjunctiva ${ }^{34-37}$ and could reduce the risk of postcataract endophthalmitis without increasing bacterial resistance. In addition to adequate povidone-iodine disinfection, we recommend combining fluoroquinolones with tobramycin as a prophylactic antibiotic, shifting from tobramycin to fluoroquinolones or other modalities such as intracameral injection at the end of surgery to prevent postoperative endophthalmitis. ${ }^{38} 39$

Multiple socioeconomic, environmental and patient factors have been associated with methicillin-resistant organisms in non-ophthalmic literature, ${ }^{40}$ however, limited investigation of risk factors has been conducted for patients with ophthalmic problem. If physicians could predict whether a patient undergoing cataract surgery is harbouring methicillin-resistant Staphylococci, they can select alternative prophylactic modalities for treatment. However, no differences in possible risk factors between methicillin-resistant and methicillin-sensitive staphylococcal groups were observed in the current study. A recent study of patients undergoing cataract surgery found that age was associated with colonisation by methicillin-resistant organisms, whereas healthcare work was unassociated. ${ }^{24}$ Hsu et al indicated that antibiotic use within the previous 30 days was a significant risk factor for colonisation by a methicillin-resistant organism, ${ }^{17}$ but we excluded the patients who used antibiotics in our study. The variable results in these studies are likely attributable to different sample sizes and different populations. Additional studies are required to establish the risk factors for colonisation by methicillin-resistant organisms.

There are certain limitations to this study. Many of the risk factors were self-reported. Nasal swabs were cultured for $S$. aureus only, because the original study protocol focused on $S$. aureus. Small sample sizes may limit statistical significance. In vitro susceptibility based on serum systemic standards does not always correlate with clinical response because no susceptibility standards are implemented for topical therapies. Finally, because the results of this study are specific to the Taiwanese environment, the findings should not be generalised to other regions or populations.

In conclusion, our study revealed that $35 \%$ of staphylococcal isolates from the conjunctiva of patients undergoing cataract were methicillin-resistant. While most Staphylococci were susceptible to fluoroquinolones, methicillin-resistant Staphylococci were more resistant than their methicillin-sensitive counterparts to tobramycin, the most common prophylactic antibiotic in Taiwan. We hope that clinicians in Taiwan consider the implications of these findings to their current clinical practice.

Acknowledgements The authors thank Mr. Lin Yu-Jr in the Biostatistical Center for ClinicalResearch, Chang Gung Memorial Hospital, Taiwan for the assistance in statistical analyses. 
Contributors $\mathrm{CHH}$ : responsible for conception and design of the study. $\mathrm{CHH}$ si and $\mathrm{CHH}$ : responsible for provision of patients. YHL and YCK: involved in collection and statistical analysis of data. LYH was involved in writing of the manuscript. YCH and $\mathrm{CHH}$ : responsible for critical revision of the manuscript. YCH, CJC, JCS, PHH and $\mathrm{CHH}$ : responsible for obtaining funding for the study. All authors: approved the final draft of the manuscript.

Funding This work was supported by National Science Council, Taiwan (NSC1032314-B-182A-043-MY2, NMRPG3D6071) and Chang Gung Memorial Hospital, Taiwan (CMRPG3F0111). The sponsor or funding organization had no role in the design or conduct of this research.

Competing interests None declared.

Ethics approval The Institutional Review Board of Chang Gung Memorial Hospital.

Provenance and peer review Not commissioned; externally peer reviewed.

Data sharing statement № additional data are available.

Open Access This is an Open Access article distributed in accordance with the terms of the Creative Commons Attribution (CC BY 4.0) license, which permits others to distribute, remix, adapt and build upon this work, for commercial use, provided the original work is properly cited. See: http://creativecommons.org/ licenses/by/4.0/

(c) Article author(s) (or their employer(s) unless otherwise stated in the text of the article) 2017. All rights reserved. No commercial use is permitted unless otherwise expressly granted.

\section{REFERENCES}

1. Callegan MC, Engelbert M, Parke DW, et al. Bacterial endophthalmitis: epidemiology, therapeutics, and bacterium-host interactions. Clin Microbiol Rev 2002;15:111-24.

2. Bannerman TL, Rhoden DL, McAllister SK, et al. The source of coagulase-negative staphylococci in the Endophthalmitis Vitrectomy Study. A comparison of eyelid and intraocular isolates using pulsedfield gel electrophoresis. Arch Ophthalmol 1997;115:357-61.

3. Morrissey I, Burnett R, Viljoen L, et al. Surveillance of the susceptibility of ocular bacterial pathogens to the fluoroquinolone gatifloxacin and other antimicrobials in Europe during 2001/2002. J Infect 2004;49:109-14

4. Speaker MG, Milch FA, Shah MK, et al. Role of external bacterial flora in the pathogenesis of acute postoperative endophthalmitis. Ophthalmology 1991;98:639-50.

5. Chung JL, Seo KY, Yong DE, et al. Antibiotic susceptibility of conjunctival bacterial isolates from refractive surgery patients. Ophthalmology 2009;116:1067-74.

6. Asbell PA, Colby KA, Deng S, et al. Ocular TRUST: nationwide antimicrobial susceptibility patterns in ocular isolates. $A m ~ J$ Ophthalmol 2008;145:951-8.

7. McDonald M, Blondeau JM. Emerging antibiotic resistance in ocular infections and the role of fluoroquinolones. J Cataract Refract Surg 2010;36:1588-98.

8. Deramo VA, Lai JC, Winokur J, et al. Visual outcome and bacterial sensitivity after methicillin-resistant Staphylococcus aureus-associated acute endophthalmitis. Am J Ophthalmol 2008;145:413-7.

9. Major JC, Engelbert M, Flynn HW, et al. Staphylococcus aureus endophthalmitis: antibiotic susceptibilities, methicillin resistance, and clinical outcomes. Am J Ophthalmol 2010;149:278-83.

10. Gentile RC, Shukla S, Shah M, et al. Microbiological spectrum and antibiotic sensitivity in endophthalmitis: a 25-year review. Ophthalmology 2014;121:1634-42.

11. Chiquet C, Maurin M, Altayrac J, et al. Correlation between clinical data and antibiotic resistance in coagulase-negative Staphylococcus species isolated from 68 patients with acute post-cataract endophthalmitis. Clin Microbiol Infect 2015:21:592 e1-592 e8.

12. von Eiff $\mathrm{C}$, Becker $\mathrm{K}$, Machka $\mathrm{K}$, et al. Nasal carriage as a source of Staphylococcus aureus bacteremia. Study Group. N Engl J Med 2001:344:11-16.

13. Ellis MW, Hospenthal DR, Dooley DP, et al. Natural history of community-acquired methicillin-resistant Staphylococcus aureus colonization and infection in soldiers. Clin Infect Dis 2004;39:971-9.

14. Chang DF, Braga-Mele R, Henderson BA, et al. Antibiotic prophylaxis of postoperative endophthalmitis after cataract surgery: results of the 2014 ASCRS member survey. J Cataract Refract Surg 2015;41:1300-5.

15. Fernández-Rubio ME, Cuesta-Rodríguez T, Urcelay-Segura JL, et al. Pathogenic conjunctival bacteria associated with systemic co-morbidities of patients undergoing cataract surgery. Eye 2013;27:915-23.

16. Hsu HY, Lind JT, Tseng L, et al. Ocular Flora and their antibiotic resistance patterns in the midwest: a prospective study of patients undergoing cataract surgery. Am J Ophthalmol 2013;155:36-44.

17. Hsu HY, Lind JT, Miller D, et al. Assessment of risk factors for oxacillin-resistant ocular flora in eyes having cataract surgery. $J$ Cataract Refract Surg 2015;41:387-92.

18. Ta CN, Chang RT, Singh K, et al. Antibiotic resistance patterns of ocular bacterial flora: a prospective study of patients undergoing anterior segment surgery. Ophthalmology 2003;110:1946-51.

19. Chen SY, Kuo MC, Wang PN, et al. Study of conjunctival flora in patients after peripheral blood stem cell transplantation and its correlation with tear secretion. Biomed J 2012;35:493-9.

20. Kim SJ, Toma HS, Midha NK, et al. Antibiotic resistance of conjunctiva and nasopharynx evaluation study: a prospective study of patients undergoing intravitreal injections. Ophthalmology 2010;117:2372-8.

21. Alabiad CR, Miller D, Schiffman JC, et al. Antimicrobial resistance profiles of ocular and nasal flora in patients undergoing intravitreal injections. Am J Ophthalmol 2011;152:999-1004.

22. Chen CJ, Wang SC, Chang HY, et al. Longitudinal analysis of methicillin-resistant and methicillin-susceptible Staphylococcus aureus carriage in healthy adolescents. J Clin Microbiol 2013;51:2508-14.

23. Kitazawa K, Sotozono $\mathrm{C}$, Sakamoto M, et al. Nasal and conjunctival screening prior to refractive surgery: an observational and crosssectional study. BMJ Open 2016;6:e010733.

24. Olson R, Donnenfeld E, Bucci FA, et al. Methicillin resistance of Staphylococcus species among health care and nonhealth care workers undergoing cataract surgery. Clin Ophthalmol 2010;4:1505-14.

25. Kunimoto DY, Das T, Sharma S, et al. Microbiologic spectrum and susceptibility of isolates: part I. postoperative endophthalmitis. Endophthalmitis Research Group. Am J Ophthalmol 1999;128:240-2.

26. Benz MS, Scott IU, Flynn HW, et al. Endophthalmitis isolates and antibiotic sensitivities: a 6-year review of culture-proven cases. Am J Ophthalmol 2004;137:38-42.

27. Watanabe K, Numata-Watanabe K, Hayasaka S. Methicillin-resistant staphylococci and ofloxacin-resistant bacteria from clinically healthy conjunctivas. Ophthalmic Res 2001;33:136-9.

28. Miño de Kaspar H, Koss MJ, He L, et al. Antibiotic susceptibility of preoperative normal conjunctival Bacteria. Am J Ophthalmol 2005;139:730-3.

29. Hori Y, Maeda N, Sakamoto M, et al. Fluoroquinolone-resistant bacteria and methicillin-resistant Staphylococci from normal preoperative conjunctiva. J Cataract Refract Surg 2008;34:711-2.

30. Hori Y, Nakazawa T, Maeda N, et al. Susceptibility comparisons of normal preoperative conjunctival bacteria to fluoroquinolones. $J$ Cataract Refract Surg 2009;35:475-9.

31. Ta CN, He L, Mino de Kaspar H. In vitro antibiotic susceptibility of preoperative normal conjunctival bacteria. Eye (Lond) 2009;23:559-60.

32. Moss JM, Sanislo SR, Ta CN. Antibiotic susceptibility patterns of ocular bacterial flora in patients undergoing intravitreal injections. Ophthalmology 2010;117:2141-5.

33. Asbell PA, Sanfilippo CM, Pillar CM, et al. Antibiotic resistance among ocular pathogens in the United States: five-year results from the antibiotic resistance monitoring in ocular microorganisms (ARMOR) Surveillance Study. JAMA Ophthalmol 2015;133:1445-54.

34. Carrim ZI, Mackie G, Gallacher G, et al. The efficacy of $5 \%$ povidoneiodine for 3 minutes prior to cataract surgery. Eur J Ophthalmol 2009;19:560-4.

35. Ciulla TA, Starr MB, Masket S. Bacterial endophthalmitis prophylaxis for cataract surgery: an evidence-based update. Ophthalmology 2002;109:13-24.

36. Darouiche RO, Wall MJ, Itani KM, et al. Chlorhexidine-Alcohol versus Povidone-lodine for Surgical-Site Antisepsis. N Engl J Med 2010;362:18-26.

37. Inoue $\mathrm{Y}$, Usui M, Ohashi $\mathrm{Y}$, et al. . Preoperative disinfection of the conjunctival sac with antibiotics and iodine compounds: a prospective randomized multicenter study. Jpn J Ophthalmol 2008;52:151-61.

38. Barry P, Seal DV, Gettinby G, et al. ESCRS study of prophylaxis of postoperative endophthalmitis after cataract surgery: preliminary report of principal results from a European multicenter study. $J$ Cataract Refract Surg 2006;32:407-10.

39. Fintelmann RE, Naseri A. Prophylaxis of postoperative endophthalmitis following cataract surgery: current status and future directions. Drugs 2010;70:1395-409. 
40. Davis SL, Perri MB, Donabedian SM, et al. Epidemiology and outcomes of community-associated methicillin- resistant Staphylococcus aureus infection. J Clin Microbiol 2007;45:1705-11. 